\title{
Rotura diafragmática traumática con herniación esplénica. Un caso inusual de hemotórax
}

\author{
Traumatic rupture of diaphragm with splenic herniation. An unusual cause of hemothorax
}

Cynthia M. González-Cantút,2*, Edson R. Marcos-Ramírez',2, Daniel Gómez-Padilla², Francisco J. Palacios-Luna ${ }^{1,2}$, Daniel A. Saldívar-Reyes ${ }^{1,2}$ y Gerardo E. Muñoz-Maldonado, ${ }^{1,2}$

${ }^{1}$ Servicio de Cirugía General, Hospital Universitario "Dr. José Eleuterio González"; ${ }^{2}$ Facultad de Medicina, Universidad Autónoma de Nuevo León, Monterrey, Nuevo León, México

\section{Resumen}

La hernia diafragmática traumática es poco frecuente en la clínica y representa un reto diagnóstico, ya que su aparición es principalmente de emergencia y esto propicia poco tiempo para la realización de estudios de imagen complementarios. Presentamos el caso de un varón de 21 años que sufrió una herida penetrante en el hemitórax izquierdo con sospecha de herniación traumática diafragmática de vísceras abdominales al tórax por radiografía, y en el quirófano se confirmó una herniación esplénica con hemorragia intratorácica. Se propone un algoritmo de manejo en estos casos tan desafiantes.

Palabras clave: Hernia diafragmática. Hernia esplénica. Rotura diafragmática. Hemotórax.

\begin{abstract}
The traumatic diaphragmatic hernia is considered a rare clinic condition and represents a diagnostic challenge. Its presentation is mainly a surgical emergency, leading to scarce time for complementary image studies. We present a case of a 21-year-old male who suffered a penetrating wound in the left hemitorax from whom is suspected to have a traumatic visceral herniation into the thoracic cavity by chest $X$-ray, confirming a splenic herniation with intrathoracic hemorrhage, as well as the proposal of a management algorithm for this defiant cases.
\end{abstract}

Key words: Diaphragmatic hernia. Splenic hernia. Diaphragmatic rupture. Hemothorax.

\section{Introducción}

Excluyendo la hernia congénita, la causa más común de hernia diafragmática en los adultos es el trauma, y los mecanismos habituales son los traumas contusos y las lesiones penetrantes ${ }^{1}$.
Dentro de la etiología traumática, el hemidiafragma izquierdo es el más afectado, reportándose su frecuencia en un $50 \%$ a $80 \%$ de los casos; las lesiones de lado derecho oscilan entre el $12 \%$ y el $40 \%$, y de forma menos común, la lesión bilateral se presenta solo en un $2 \%$ de los casos ${ }^{2}$. Esta predominancia se

\section{Correspondencia:}

*Cynthia M. González-Cantú

Avda. Madero s/n

Col. Mitras Centro

Fecha de recepción: 26-11-2020

C.P. 64460 , Monterrey, N.L., México

E-mail: dracynthiacantu@gmail.com

0009-7411/0 2020 Academia Mexic

(http://creativecommons.org/licenses/by-nc-nd/4.0/).
Fecha de aceptación: 26-12-2020

DOI: 10.24875/CIRU.20001304
Cir Cir. 2021;89(S1):82-86

Contents available at PubMed www.cirugiaycirujanos.com ajo la licencia CC BY-NC-ND 
debe a la debilidad congénita de la fusión de la región costal y lumbar del hemidiafragma izquierdo, así como a la posición anatómica protectora del hígado 3 .

La rotura diafragmática es un predictor de lesión grave; hasta el $6 \%$ están asociadas con lesiones torácicas y el $22 \%$ con lesiones abdominales ${ }^{4}$. Su diagnóstico es complejo y el retraso en establecerlo conlleva una mayor morbimortalidad ${ }^{5}$. En el escenario donde los órganos abdominales herniados están además lesionados, puede presentarse una hemorragia intratorácica que lleve a una verdadera situación de emergencia ${ }^{6}$.

Presentamos nuestra experiencia en el manejo de un paciente con herniación esplénica y hemorragia intratorácica.

\section{Caso clínico}

Varón de 21 años sin antecedentes médicos relevantes que es trasladado al servicio de urgencias del hospital 30 minutos después de sufrir una herida por arma punzocortante en el sexto espacio intercostal, en la línea axilar anterior, del hemitórax izquierdo, con una longitud aproximada de $4 \mathrm{~cm}$ y con penetración a la cavidad torácica (Fig. 1).

A su ingreso se valora al paciente taquicárdico, presión arterial 100/60 $\mathrm{mmHg}$ y saturación de oxígeno del $96 \%$ al aire ambiente. Se aplica el Protocolo Avanzado para Trauma para valoración y se canalizan dos vías venosas de alto calibre para iniciar reanimación hidroelectrolítica. En la exploración torácica se visualiza un tórax simétrico con disminución de los movimientos respiratorios del hemitórax izquierdo; a la auscultación, los ruidos respiratorios en el hemitórax izquierdo no son audibles comparados con los del hemitórax derecho, y los ruidos cardiacos sin alteraciones; a la percusión del tórax se evidencia un sonido mate en el hemitórax izquierdo. No se aprecian desviación de la tráquea ni presencia de distención yugular en el cuello. Debido a que clínicamente se pudo apreciar un síndrome pleuropulmonar en la exploración torácica, se decide colocar una sonda de toracostomía izquierda, obteniendo salida de aire y sangre de aproximadamente $700 \mathrm{ml}$. El paciente se mantiene hemodinámicamente estable posterior a la colocación del drenaje torácico. En la exploración abdominal presenta dolor a la palpación con predominio en los cuadrantes superiores. El resto de la exploración física no ofrece datos de interés. Se completan exámenes de laboratorio, y en la radiografía de tórax se observa el hemidiafragma izquierdo elevado

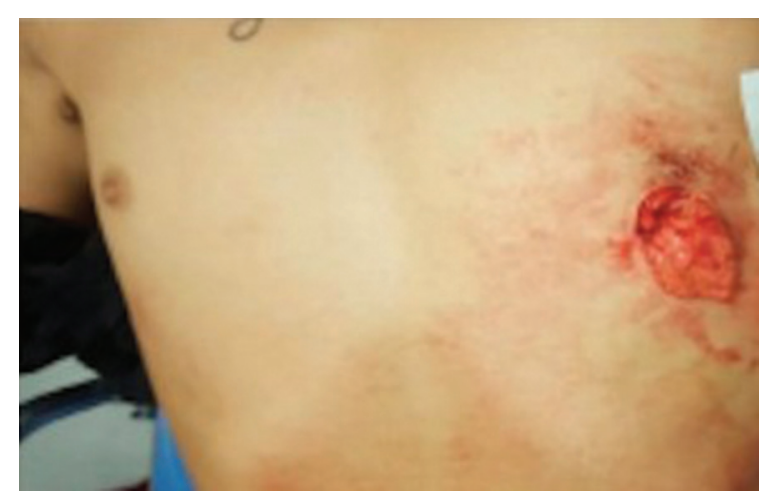

Figura 1. Herida penetrante en el tórax a nivel de la línea axilar anterior en el sexto espacio intercostal.

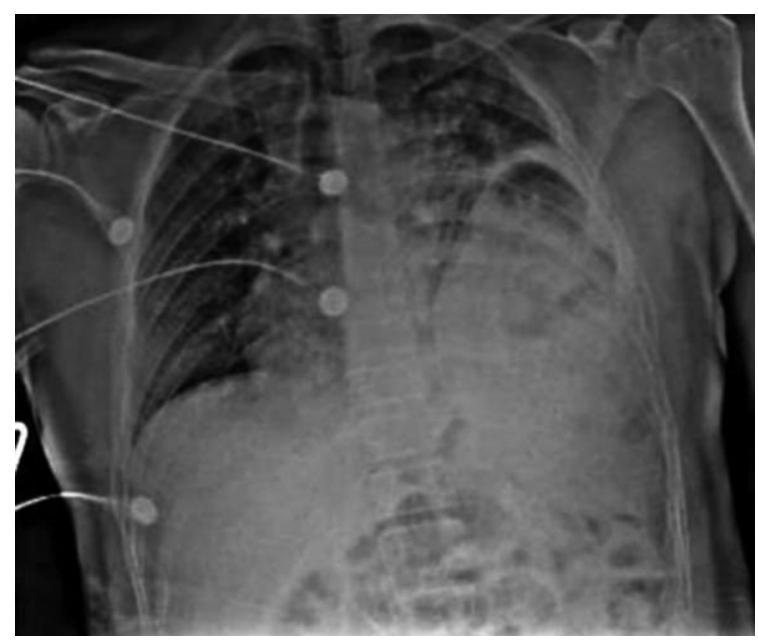

Figura 2. Radiografía de tórax preoperatoria en la que se identifica la presencia de una hernia diafragmática traumática izquierda.

con presencia de la burbuja gástrica en el hemitórax izquierdo, imagen sugestiva de herniación diafragmática traumática, por lo que se decide pasar de emergencia al quirófano (Fig. 2).

Se realiza laparotomía exploratoria y se encuentra hemoperitoneo de aproximadamente $300 \mathrm{ml}$ en corredera izquierda, así como herniación de fondo y cuerpo gástrico, ligamento gastroesplénico y bazo a través de una lesión de $7 \mathrm{~cm}$ de longitud y aproximadamente $4 \mathrm{~cm}$ de ancho en el domo izquierdo del diafragma (Fig. 3). Se realiza el retorno de las vísceras al abdomen y se identifica una lesión esplénica de grado IV, por lo que se procede a realizar esplenectomía, así como una lesión hepática de grado II de aproximadamente $5 \mathrm{~cm}$ en el lóbulo hepático izquierdo, en los segmentos laterales superior e inferior (Couinaud 2 y 3), y se realiza hemostasia hepática. En el 


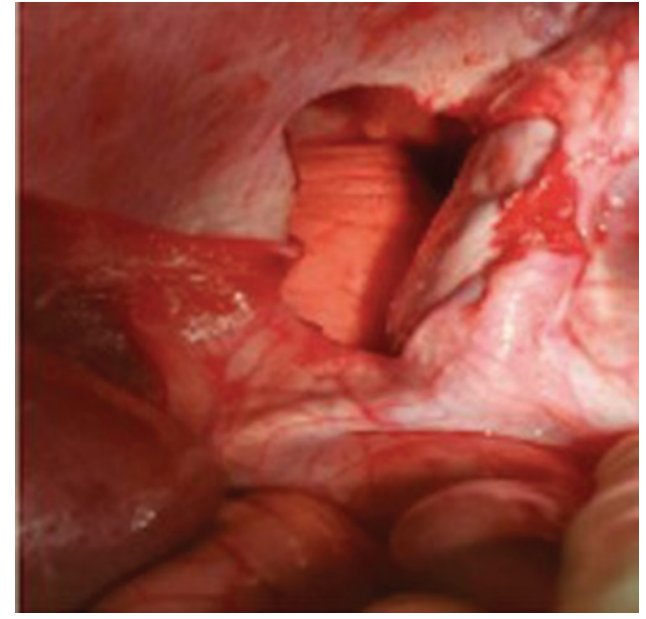

Figura 3. Imagen en el transoperatorio que muestra el defecto diafragmático.

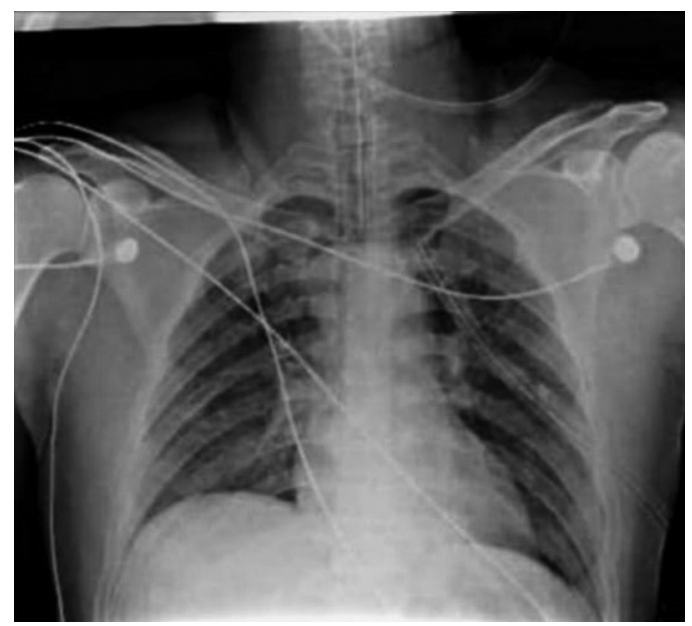

Figura 4. Radiografía de tórax posoperatoria que evidencia la reparación diafragmática con reducción de la hernia.

resto de la exploración quirúrgica no se evidencia lesión gástrica ni ninguna otra lesión en los órganos intraabdominales.

A través de la amplia lesión diafragmática se pudo advertir una lesión de $2 \mathrm{~cm}$ en el lóbulo inferior del pulmón izquierdo, por lo cual se realiza neumorrafia ininterrumpida con Vicry ${ }^{\circledR}$ 4-0. Se realiza evacuación de coágulos y lavado en la cavidad torácica, así como confirmación de la correcta posición y adecuado funcionamiento de la sonda de toracostomía, y posterior frenorrafia con Vicryl ${ }^{\circledR}$ 3-0. A continuación se procede a lavado exhaustivo de la cavidad abdominal, verificación de la hemostasia y colocación de un drenaje cerrado en la corredera izquierda. En la figura 4 se muestra el resultado radiológico de la cirugía.
El paciente pasa a recuperación y posteriormente a sala de observación, cursando un posoperatorio sin eventualidades. En el cuarto día de posoperatorio se retira el drenaje abdominal y un día después, descartando la presencia de fístula pleural y con un débito de $50 \mathrm{ml}$ seroso por la sonda de toracostomía, se decide su retiro con control radiográfico, que se realiza sin complicaciones y se decide el egreso hospitalario. El paciente continúa seguimiento por la consulta y se retira la sutura del abordaje abdominal en el día 10 de posoperatorio.

\section{Discusión}

La rotura diafragmática se considera una urgencia absoluta, ya que presenta una alta mortalidad que va del $21 \%$ al $25 \%$. Su sospecha y diagnóstico oportuno han demostrado ser cruciales para la disminución de la mortalidad, pero solo en el $20 \%$ de los casos es posible ${ }^{8}$.

La rotura puede ir acompañada de la herniación de órganos de la cavidad abdominal hacia el tórax debido al gradiente de presión pleuroperitoneal. Entre los órganos que con más frecuencia se hernian se encuentran el estómago, el epiplón, el intestino delgado, el colon y el bazo ${ }^{9}$.

Ante una rotura diafragmática con hemotórax se deben realizar manejo y resucitación inicial del paciente politraumatizado acorde con el protocolo ATLS $^{\circledR}$. El paciente deberá tener accesos intravenosos de gran calibre, monitorización cardiaca y de oxígeno continua, y registro del electrocardiograma en 12 derivaciones. Las lesiones inmediatas que ponen en peligro la vida requerirán una intervención urgente, como la toracostomía ${ }^{6}$.

La radiografía simple es el estudio de imagen de inicio en los casos de hernia intermitente o en la fase temprana de una rotura diafragmática. En estos casos, solo en un $25-30 \%$ de las radiografías se encuentran hallazgos anormales, entre ellos elevación del hemidiafragma involucrado en la lesión, aplanamiento del ángulo costodiafragmático o presencia de hemo-neumotórax. En los casos en que los defectos son poco extensos se ha reportado un mayor número de falsos negativos ${ }^{5,9}$.

De forma clásica, ante una cirugía de urgencia que compromete órganos abdominales se realiza una laparotomía exploratoria por medio de una incisión media abdominal. La extensión y la localización de la herida dependerán de la preferencia del cirujano, de los órganos que se sospecha que están involucrados 
en la lesión y de los hallazgos transoperatorios, para permitir una completa exploración y manejo ${ }^{10}$. Otra opción de abordaje es la laparoscópica, pero se reserva para situaciones no urgentes, como la reparación de secuelas, y para el cirujano con experiencia en mínima invasión ${ }^{11}$.

En el presente caso se brindaron las medidas de resucitación recomendadas por el ATLS $^{\circledR}$. Debido a la ausencia de ruidos respiratorios en el hemidiafragma homolateral a la lesión, se decidió colocar una sonda de toracostomía en la sala de resucitación, aunado a la exploración física (palpación dolorosa de los cuadrantes superiores del abdomen) y los estudios de imagen (radiografía de tórax que evidenció la herniación de contenido abdominal en el hemitórax izquierdo) que fortalecieron la sospecha diagnóstica, por lo cual se decidió realizar una laparotomía de emergencia.

En nuestro paciente se evidenció una herida por arma punzocortante a nivel del sexto espacio intercostal, línea axilar anterior, con lesión de $7 \mathrm{~cm}$ de longitud en el domo izquierdo del diafragma, lesión de $2 \mathrm{~cm}$ en el lóbulo inferior del pulmón izquierdo, lesión esplénica y lesión hepática de grado II de aproximadamente $5 \mathrm{~cm}$ en el lóbulo izquierdo, en los segmentos laterales superior e inferior. El tamaño del defecto diafragmático fue lo suficientemente extenso para exponer el trayecto de la lesión, la cual era única y se encontraba cerca del límite de las cavidades torácica y abdominal, por lo cual no se decidió realizar un abordaje de toracotomía.

Tras la identificación de una lesión diafragmática, con presencia o no de una hernia, debe ser reparada de forma inmediata. Todo órgano abdominal presente en la cavidad pleural deberá ser reducido y reubicado en su localización original. Posterior a la reducción de la hernia se debe realizar desbridamiento de los bordes necróticos presentes en la lesión para un correcto cierre del defecto, especialmente cuando la lesión se ha producido por un proyectil de fuego ${ }^{12}$. En caso de contaminación masiva por contenido gastrointestinal en la cavidad pleural se debe llevar a cabo una toracotomía con lavado profuso y colocación de sonda endopleural para drenaje continuo de secreciones ${ }^{13}$.

Los órganos abdominales deben ser regresados a la cavidad abdominal y explorados en busca de lesiones. En nuestro manejo se realizó esplenectomía debido a la lesión extensa sobre el hilio vascular, se realizó también hemostasia hepática de los segmentos laterales superior e inferior, y se exploró la cavidad en busca de otras probables lesiones asociadas, tales como lesión de colon transverso o descendente, o lesión gástrica debido a la dirección del impacto. Se realizó un lavado exhaustivo de la cavidad abdominal y se decidió la colocación de un drenaje cerrado dirigido a la fosa esplénica. Actualmente no existe un estándar descrito sobre la colocación o no de drenajes posterior a la esplenectomía, y mayormente la elección es a preferencia del cirujano ${ }^{14,15}$.

Posterior a la reparación diafragmática es posible encontrar complicaciones como la dehiscencia; la principal causa predisponente a esta es la realización de un cierre bajo tensión. Cuando se realiza la valoración del defecto desde el momento quirúrgico se debe contemplar, dependiendo de las dimensiones de este, si el cierre es libre de tensión y podrá ser realizado. En estos casos es recomendable la colocación de algún parche pequeño de epiplón para permitir un cierre sin tensión, ya que el riesgo de recidiva es alto al realizar el cierre de esta manera, debido a que durante la cirugía, bajo la anestesia, las presiones presentes en el diafragma son menores que las normales y estas se incrementan durante la bipedestación y la maniobra de Valsalva, escenarios que ocurren durante el posoperatorio y se deben tener en consideración ${ }^{16}$.

El cirujano debe estar familiarizado con las diversas alternativas para realizar la reparación diafragmática. En caso de no poder realizar el cierre primario, estas opciones varían entre reconstrucciones con tejido autólogo o con aloplásticos ${ }^{16-21}$. Recientemente se han descrito nuevos materiales biológicos aptos para su uso en la reconstrucción ${ }^{22,23}$.

Es de importancia señalar que la tasa más alta de dehiscencia posterior a la colocación de estos materiales se presenta en el receso costofrénico posterior izquierdo, en el cual se han reportado en la literatura incidencias tan altas como del $5 \%$ al $7.5 \%{ }^{24}$.

En el presente caso se logró realizar el cierre primario del defecto diafragmático obteniendo un cierre libre de tensión, sin presentar dehiscencia durante su evolución.

En cuanto a las lesiones del lóbulo pulmonar, se han descrito reparaciones con sutura, engrapadora, clamps y la resección no anatómica. En nuestro paciente se realizó una neumorrafia con surgete continuo 2-0. Una de las posibles complicaciones posterior a estas lesiones es la fístula broncopleural residual, la cual sería demostrada en el Pleurovac ${ }^{\circledR}$ al momento de la evaluación dinámica inspiratoria y espiratoria. También es de suma importancia en este caso la evolución del gasto torácico, pasando de ser hemático franco a 
serohemático y a disminuir en cantidad. Si el drenaje no presentara un descenso ni un cambio de calidad, nos haría pensar en la permanencia de la lesión o la presencia de alguna lesión inadvertida, como en el caso de las lesiones de las arterias intercostales con la colocación o la movilización de la sonda de toracostomía $25-28$.

\section{Conclusiones}

La rotura diafragmática representa una urgencia quirúrgica absoluta, pues tiene una alta tasa de mortalidad. El diagnóstico preoperatorio solo es posible en el $20 \%$ de los casos, lo cual reitera la importancia de la sospecha clínica durante la evaluación inicial del trauma.

$\mathrm{Ni}$ la cinemática del traumatismo ni la presencia o ausencia de herniación visceral incrementarán la tasa de mortalidad tal como lo hace la demora diagnóstica.

La presencia de hemotórax debido a la lesión de órganos herniados a la cavidad torácica es inusual, siendo prioritaria su resolución quirúrgica inmediata.

\section{Agradecimientos}

Nuestro eterno agradecimiento a los pacientes, cuyo bienestar es el objetivo de nuestra prática diaria.

\section{Responsabilidades éticas}

Protección de personas y animales. Los autores declaran que para esta investigación no se han realizado experimentos en seres humanos ni en animales.

Confidencialidad de los datos. Los autores declaran que han seguido los protocolos de su centro de trabajo sobre la publicación de datos de pacientes.

Derecho a la privacidad y consentimiento informado. Los autores han obtenido el consentimiento informado de los pacientes y/o sujetos referidos en el artículo. Este documento obra en poder del autor de correspondencia.

\section{Financiamiento}

El financiamiento fue brindado por recursos propios del Servicio de Cirugía General del Hospital Universitario. No se contó con apoyo de ninguna otra institución o casa distribuidora.

\section{Conflictos de intereses}

Todos los autores declaran no tener conflicto de intereses.

\section{Bibliografía}

1. Vinnicombe Z, Little $M$, Wan A. An unusual diaphragmatic hernia with gastric perforation and sub-acute presentation. Ann R Coll Surg Engl. 2016;98:e181-e383

2. Gonz I, Soto E, Villanueva E. Ruptura diafragmática traumática diagnosticada preoperatoriamente. A propósito de un caso. An Sist Sanit Navar. 2010;33:97-101

3. Serra Valdés MA, Achon Polhamus M, Menéndez Villa M de L, Carnesoltas Suárez L. Traumatic diaphragmatic hernia: case report. Medwave. 2013;13:e5636.

4. George L, Rehman SU, Khan FA. Diaphragmatic rupture: a complication of violent cough. Chest. 2000;117:1200-1.

5. Meyers BF, McCabe CJ. Traumatic diaphragmatic hernia: occult marker of serious injury. Ann Surg. 1993;218:783-90.

6. Dennis BM, Gondek SP, Guyer RA, Hamblin SE, Gunter OL, Guillamondegui OD. Use of an evidence-based algorithm for patients with traumatic hemothorax reduces need for additional interventions. J Trauma Acute Care Surg. 2017:82:728-32.

7. Düzgün AP, Özmen MM, Saylam B, Coşkun F. Factors influencing mortality in traumatic ruptures of diaphragm. Ulus Travma ve Acil Cerrahi Derg. 2008;14:132-8.

8. Hanna WC, Ferri LE, Fata P, Razek T, Mulder DS. The current status of traumatic diaphragmatic injury: lessons learned from 105 patients over 13 years. Ann Thorac Surg. 2008;85:1044-8.

9. Eren S, Çiriş F. Diaphragmatic hernia: diagnostic approaches with review of the literature. Eur J Radiol. 2005;54:448-59.

10. Burger JWA, Van 't Riet M, Jeekel J. Abdominal incisions: techniques and postoperative complications. Scand J Surg. 2002:91:315-21.

11. Ellis $\mathrm{H}$. Applied anatomy of abdominal incisions. Br J Hosp Med. 2010;71(3 Suppl.):36-7.

12. Petrone $P$, Asensio JA, Marini $C P$. Diaphragmatic injuries and post-traumatic diaphragmatic hernias. Curr Probl Surg. 2017;54:11-32.

13. Carter R, Brewer LA. Strangulating diaphragmatic hernia. Ann Thorac Surg. 1971:12:281-90

14. Carmichael J, Thomas WO, Dillard D, Luterman A, Ferrara JJ. Indications for placement of drains in the splenic fossa. Am Surg. 1990;56: 313-8.

15. Vecchio R, Intagliata E, Marchese S, Battaglia S, Cacciola RR, Cacciola E. Surgical drain after open or laparoscopic splenectomy: is it needed or contraindicated? G Chir. 2015:36:101-5.

16. Solli $P$, Bertolaccini L, Brandolini J, Pardolesi A. Reconstructive techniques after diaphragm resection and use of the diaphragmatic flap in thoracic surgery. Shanghai Chest. 2017;1:21.

17. Bedini AV, Andreani SM, Muscolino G. Latissimus dorsi reverse flap to substitute the diaphragm after extrapleural pneumonectomy. Ann Thorac Surg. 2000;69:986-8.

18. Suzuki K, Takahashi T, Itou Y, Asai K, Shimota H, Kazui T. Reconstruction of diaphragm using autologous fascia lata: an experimental study in dogs. Ann Thorac Surg. 2002;74:209-12.

19. Koot VCM, Bergmeijer JH, Molenaar JC. Lyophylized dura patch repair of congenital diaphragmatic hernia: occurrence of relapses. J Pediatr Surg. 1993;28:667-8.

20. Rolli L, Leuzzi G, Girotti P, Duranti L, Pastorino U. Permeable nonabsorbable mesh for total diaphragmatic replacement in extended pneumonectomy. Ann Thorac Surg. 2017; 104:e105-7.

21. Schiavon M, De Filippis A, Marulli G, Rea F. A new technique of diaphragmatic patch fixation in extrapleural pneumonectomy. Eur J Cardiothorac Surg. 2010;38:798-800.

22. Al-Nouri O, Hartman B, Freedman R, Thomas C, Esposito T. Diaphragmatic rupture: is management with biological mesh feasible? Int J Surg Case Rep. 2012;3:349-53.

23. Butler CE, Prieto VG. Reduction of adhesions with composite alloderm polypropylene mesh implants for abdominal wall reconstruction. Plast Reconstr Surg. 2004;114:464-73.

24. Dasilva MC, Sugarbaker DJ. Technique of extrapleural pneumonectomy. YOTCT. 2010;15:282-93.

25. Havelock T, Teoh R, Laws D, Gleeson F. Pleural procedures and thoracic ultrasound: British Thoracic Society pleural disease guideline 2010. Thorax. 2010;65(Suppl 2).

26. York EL, Lewall DB, Hirji M, Gelfand ET, Modry DL. Endoscopic diagnosis and treatment of postoperative bronchopleural fistula. Chest. 1990:97:1390-2

27. Prado AF, Linares PM, Donoso FA, Cruces RP, Herrera OP, Contreras El. Cierre efectivo por vía broncoscópica de fístula broncopleural de alto flujo en paciente con síndrome de distress respiratorio agudo grave. Rev Chil Pediatr. 2006;77:501-5.

28. Watanabe SI, Watanabe T, Urayama H. Endobronchial occlusion method of bronchopleural fistula with metallic coils and glue. Thorac Cardiovasc Surg. 2003;51:106-8. 